\title{
AN ALTERNATIVE MATTING LAPLACIAN
}

\author{
François Pitié \\ School of Engineering \\ Trinity College Dublin
}

\begin{abstract}
Cutting out and object and estimate its transparency mask is a key task in many applications. We take on the work on closed-form matting by Levin et al.[1], that is used at the core of many matting techniques, and propose an alternative formulation that offers more flexible controls over the matting priors. We also show that this new approach is efficient at upscaling transparency maps from coarse estimates.
\end{abstract}

Index Terms - Image segmentation, Matting

\section{INTRODUCTION}

Pulling a matte from a film or video sequence is the act of cutting out an object from its background by creating a transparency mask or $\alpha$ matte, that ranges between 0 and 1 . An $\alpha$ value of 0 at pixel $i$ means that only the background colour $B_{i}$ is visible, an $\alpha$ value of 1 means that only the foreground colour $F_{i}$ is visible, and values in-between 0 and 1 manifest a blend between the object and the background:

$$
C_{i}=\alpha_{i} F_{i}+\left(1-\alpha_{i}\right) B_{i}
$$

To help the algorithms, techniques typically ask the user to scribble a trimap, defining foreground, background and the unknown region to be pulled.

Since the successful work of Chuang et al. [2] on bayesian matting in 2001, the notion of matting as an inference problem has been explored by several authors (see in particular Poisson Matting [2] and Inference Matting [3]). In 2007 Levin et al. [1] proposed a number of remarkable advances by finding a closed-form solution to the matting problem. Their solution is fast and very often used as a core component in subsequent matting techniques [3, 4, 5].

A key contribution from Levin et al. [1] is to consider that the transparency value can be approximated as a linear combination of the colour components:

$$
\alpha_{i}=a_{i}^{T} C_{i}+b_{i}
$$

with $a_{i}=\left[a_{i}^{R}, a_{i}^{G}, a_{i}^{B}\right]$. Instead of a non-linear problem with 6 unknowns ( 3 for $F, 3$ for $B$ ), we are left with a linear model

This work is supported by the SFI research centre ADAPT. http://adaptcentre.ie/ with 4 unknowns ( 3 for $a$ and 1 for $b$ ). This linear approximation is justified by the fact that colours often spread along 3D lines in the colour space (see Hillman et al. [6]).

Interestingly this approach has been used routinely in video post-production for a long time. For instance, tools used to extract transparency maps for green or blue screens (also called keyers) are often based on a simple linear fit.

The second contribution of Levin's work is to introduce a smoothness constraint that yields a global closed-form solution. They state that the matting model should hold for overlapping $3 \times 3$ image patch $w_{i}$. This leads to the following energy to minimise:

$$
J(\alpha, a, b)=\sum_{i} \sum_{j \in w_{i}}\left(a_{i}^{T} C_{j}+b_{i}-\alpha_{j}\right)^{2}+\epsilon\left\|a_{j}\right\|^{2}
$$

The term $\epsilon\left\|a_{j}\right\|^{2}$ adds further smoothness to the matte by damping $a$ towards zero and thus flattening the matte.

At this point Levin et al have chosen to integrate $a, b$ out of the problem and so generate a marginalised estimate of $\alpha$. This leads to a linear system as follows:

$$
(L+D) \alpha=D \alpha_{0}
$$

where $\alpha_{0}$ is the initial guess for the transparency values, and $D$ a diagonal matrix of the confidence values for this guess. The sparse matrix $L$ is the matting Laplacian, derived from Eq. (3), with entries as follows:

$L_{i, j}=\sum_{k \mid i, j \in w_{k}} \delta_{i, j}-\frac{1+\left(C_{i}-\mu_{k}\right)\left(R_{k}+\frac{\epsilon}{\left|w_{k}\right|}\right)^{-1}\left(C_{j}-\mu_{k}\right)}{\left|w_{k}\right|}$

where $\mu_{k}$ and $R_{k}$ are the mean and covariance of patch $w_{k}$.

Contribution. We argue in this paper that instead of integrating out $a$ and $b$, it might be more interesting to integrate out $\alpha$ and solve for $a$ and $b$ instead. In Fig. 1 is an example of our estimates for $a$ and $b$ ( $a$ has been rescaled to $a / 5+.5$ to better show the effect). We can think of $a$ as the colour of the colour filter applied to the input picture to reveal $\alpha$. Whereas the values of $\alpha$ might vary quickly and change across the image scales, the values of $a$ and $b$ are locally smooth. Levin et al. take advantage of this for upscaling $\alpha$ map without solving 


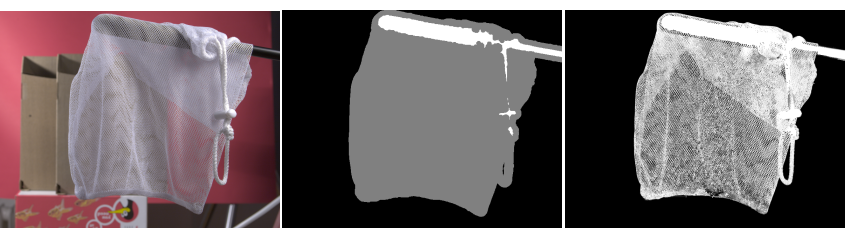

(a)

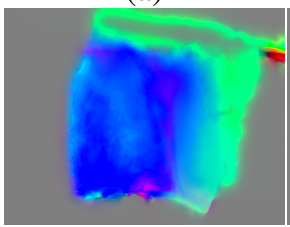

(d) (b)

(c)

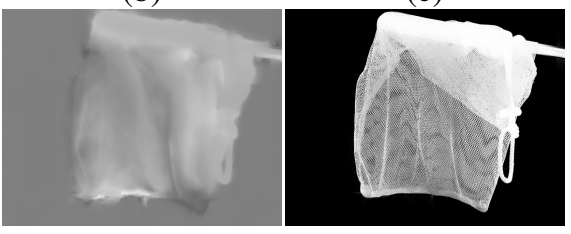

(e)

(f)

Fig. 1. Example. (a) input image, (b) trimap, (c) $\alpha_{0}$ from [3], (d) estimated $a$, (e) estimated $b$, (f) estimated $\alpha$.

the linear system. Estimates of $a$ and $b$ are derived from the lowres $\alpha$ map and a highres $\alpha$ map is obtained by applying Eq. (2) on the upscaled $a$ and $b$. What we propose is to work directly with $a$ and $b$ and avoid the estimation step from $\alpha$. This will result in a cleaner estimation of $a$ and $b$.

In closed-form matting, it is hard to finely control the scale of the spatial smoothness. The minimum stencil size for $\alpha$ is a $5 \times 5$ stencil, and larger patches require a different approach [7] to be practical. We show that working directly with $a$ and $b$ allows us to use a more compact 5-point stencil and gives us a simpler way to control the spatial smoothness.

Lastly, the matting Laplacian of Eq. (5) needs to be positive definite. In [1], this is enforced by adding the unary prior $\epsilon\|a\|^{2}$. The problem is that this prior is purely used for numerical stability and does not yield interesting matting properties. This is noted in Pitié and Kokaram [8] who argue that a better approach is to set $\epsilon$ only in the direction of small eigenvalues of the covariance matrix. Other priors on the patch covariance matrix are discussed in [9], but then again, the analysis is limited to the patch size. We argue in this paper that working with $a$ and $b$ yield in simpler and more useful priors.

Organisation of the paper. In section 2, we derive an alternate matting Laplacian formulation for $a$ and $b$. We also expose how the spatial and unary priors can be set in this new framework. Results and comparisons of our approach to closed-form matting are presented in section 3 .

\section{MATTING COLOUR FILTER}

Let us use the notation $X=[C ; 1]$ as it allows us to write the linear matting model in a slightly more compact form:

$$
\alpha_{i}=X_{i}^{T} \beta_{i}
$$

The model parameters $\beta_{i}=\left[a_{i} ; b_{i}\right]$ is of dimension $4 \times 1$ and is the concatenation of $a$ and $b$ of closed-form matting.

\subsection{Matting Laplacian for $\beta$}

We start from the same energy as in Eq. (3), but ignoring for the moment the penalty term on $\epsilon\|a\|^{2}$ and only focusing on the spatial constraints:

$$
J^{*}(\alpha, \beta)=\sum_{i} \sum_{j \in w_{i}}\left(X_{j}^{T} \beta_{i}-\alpha_{j}\right)^{2}
$$

Denote $w_{j}^{-1}=\left\{i: j \in w_{i}\right\}$ and reorder the summation:

$$
J^{*}(\alpha, \beta)=\sum_{j} \sum_{i \in w_{j}^{-1}}\left(X_{j}^{T} \beta_{i}-\alpha_{j}\right)^{2}
$$

Now let us integrate out $\alpha$ :

$$
J^{*}(\beta)=\sum_{j} \min _{\alpha_{j}} \sum_{i \in w_{j}^{-1}}\left(X_{j}^{T} \beta_{i}-\alpha_{j}\right)^{2}
$$

The least square estimate for $\alpha_{j}$ is $\frac{1}{\left|w_{j}^{-1}\right|} \sum_{k \in w_{j}^{-1}} X_{j}^{T} \beta_{k}$,

$$
\begin{gathered}
J^{*}(\beta)=\sum_{j} \sum_{i \in w_{j}^{-1}}\left(X_{j}^{T} \beta_{i}-\frac{1}{\left|w_{j}^{-1}\right|} \sum_{k \in w_{j}^{-1}} X_{j}^{T} \beta_{k}\right)^{2} \\
J^{*}(\beta)=\sum_{j} \sum_{i \in w_{j}^{-1}}\left(\frac{1}{\left|w_{j}^{-1}\right|} \sum_{k \in w_{j}^{-1}} X_{j}^{T}\left(\beta_{i}-\beta_{k}\right)\right)^{2}
\end{gathered}
$$

This is a quadratic form in $\beta$. We can rewrite it as follows:

$$
J^{*}(\beta)=\sum_{i, k} \beta_{i}^{T} A_{i, k} \beta_{k}
$$

with

$$
A_{i, k}= \begin{cases}-\sum_{j: i, k \in w_{j}^{-1}} \frac{2}{\left|w_{j}^{-1}\right|^{2}} X_{j} X_{j}^{T} & \text { if } i \neq k \\ -\sum_{l \neq i} A_{i, l} & \text { if } i=k\end{cases}
$$

Similarly to the closed-form approach, we end up with a linear system of equations:

$$
\left(A+A_{0}\right) \beta=A_{0} \beta_{0}
$$

The trimap and other priors are encoded into $A_{0}$ and $\beta_{0}$ and will be discussed in the following sections on unary priors.

This time the matting Laplacian $A=\left(A_{i, k}\right)$ is a block banded matrix, with each block matrix being of size $4 \times 4$. Importantly this linear system solves the exact same energy as in closed-form matting. Thus solving for $\beta$ and then resolving the matting equation $\alpha=x_{i}^{T} \beta_{i}$ is the same as solving directly for closed-form matting. In the following, we discuss the benefits of this approach. 


\subsection{Matting as an Anisotropic Diffusion}

In closed-form matting, the use of the inverse of the covariance matrix in Eq. (5) requires a minimum patch size to ensure numerical stability of the matrix inversion. There is no such numerical issue here, and we can safely consider much smaller patch sizes. For instance, for patches of size 2 (i.e. vertical and horizontal pairs), it follows a 5-point stencil linear system:

$$
A_{i, k}= \begin{cases}-\frac{1}{2}\left(X_{i} X_{i}^{T}+X_{k} X_{k}^{T}\right) & \text { if } i \neq k, k \in \mathcal{N}_{i} \\ -\sum_{l \neq i} A_{i, l} & \text { if } i=k\end{cases}
$$

where $\mathcal{N}_{i}$ is the 5-point stencil of pixel $i$.

Interestingly this system corresponds to an anisotropic diffusion process for $\beta$. In continuous settings, the smoothness constraint is in fact equivalent to:

$$
\operatorname{div}\left(X^{T} \nabla \beta\right)=0
$$

where the spatial gradient $\nabla \beta$ is of dimension $4 \times 2$. Note that it is possible to start from this formulation to derive Eq. (15) using finite difference approximation.

\subsection{Spatial Priors}

In closed-form matting, the prior on the spatial smoothness is controlled via a penalty energy $\epsilon\|a\|^{2}$ which biases $a$ towards zero, hence making sure that $\alpha$ is smooth. This prior is used to enable the stable inversion of the colour covariance matrix.

With our proposed formulation, there is another way of imposing spatial prior by setting a prior on $X X^{T}$. The matrix $X X^{T}$ is of rank 1 . This can make $A$ indefinite and thus unstable to solve. However, a number of simple priors on the colour distribution of $X$ can help making $A$ definite positive.

For instance, we can replace $X_{i} X_{i}^{T}$ with $X_{i} X_{i}^{T}+\epsilon_{s} I_{4}$ in Eq. (15). This increases the rank to 4 by adding a small isotropic Gaussian prior on the distribution of $X$. Alternatively, we can replace $X_{i} X_{i}^{T}$ with a local estimate of $\mathbb{E}\left\{X_{i} X_{i}^{T}\right\}$, which can be obtained by a spatial Gaussian blur (variance $\sigma_{s}$ ) of the values of $X_{i} X_{i}^{T}$.

We believe that these kind of spatial priors are more principled and useful.

\subsection{Unary Priors}

In closed-form matting, the unary priors are limited to a prior on $\alpha$ and the penalty energy $\epsilon\|a\|^{2}$. Here we explore what can be gained from using a full Multivariate Gaussian prior on $\beta$ :

$$
U_{\beta}\left(\beta_{i}\right)=\frac{1}{2}\left(\beta_{i}-\beta_{i, 0}\right)^{T} A_{i, 0}\left(\beta_{i}-\beta_{i, 0}\right)^{T}
$$

where $A_{i, 0}$ is the covariance of the of MVG prior and $\beta_{i 0}$ the expected mean value. For a practical design of $A_{i, 0}$, consider

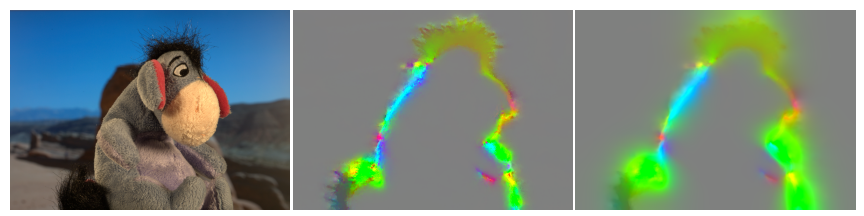

(a)

(b)

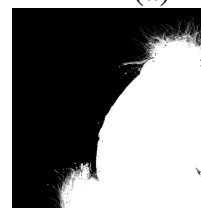

(d)

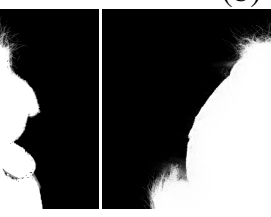

(e)

(f)

Fig. 2. Input image on (a). (b) $\beta$ estimated using closedform, (c) using our technique $\left(\sigma_{s}=1, \epsilon_{s}=10^{-4}\right)$, $\operatorname{MAD}(\mathrm{e}, \mathrm{f})=0.0025$.

that we have $N$ samples $X_{1}, \cdots, X_{N}$ for which we have expected $\alpha_{1}, \cdots, \alpha_{N}$ and confidence measure $\lambda_{1}, \cdots, \lambda_{N}$ :

$$
\begin{aligned}
& U_{\beta}\left(\beta_{i}\right)=\frac{1}{2 N} \sum_{n=1}^{N} \lambda_{n}\left(X_{n}^{T} \beta_{i}-\alpha_{n}\right)^{2} \\
& =\frac{1}{2 N} \sum_{n=1}^{N} \lambda_{n} \beta_{i}^{T} X_{n} X_{n}^{T} \beta_{i}+\alpha_{n}^{2}-2 \lambda_{n} \alpha_{n} X_{n}^{T} \beta_{i} \\
& =\frac{1}{2}\left(\beta_{i}-A_{i, 0}^{-1} \mu_{i, 0}\right)^{T} A_{i, 0}\left(\beta_{i}-A_{i, 0}^{-1} \mu_{i, 0}\right)
\end{aligned}
$$

with $A_{i, 0}=\frac{1}{N} \sum_{n=1}^{N} \lambda_{n} X_{n} X_{n}^{T}$ and $A_{i, 0} \beta_{i, 0}=\mu_{i, 0}=$ $\frac{1}{N} \sum_{n=1}^{N} \lambda_{n} \alpha_{n} X_{n}$. The complete linear system is as follows:

$$
\left(A+A_{0}\right) \beta=\mu_{0}
$$

where $A_{0}$ is a block diagonal made up of all the $A_{i, 0}$ matrices and vector $\mu_{0}=A_{i, 0} \beta_{0}$ is the concatenation of all $\mu_{0, i}$.

Prior on $\alpha$. If we want to for $\alpha_{i}$ to be closed to $\alpha_{i 0}$, then we can set $A_{i, 0}=\lambda X_{i} X_{i}^{T}$ and $\mu_{\alpha X}=\lambda \alpha_{i 0} X_{i}$.

Prior on $F$. For a prior on $X_{F}$, then we set $A_{i, 0}=\lambda X_{F} X_{F}^{T}$ and $\mu_{i, 0}=\lambda X_{F}$. Alternatively, if we have a distribution for $X_{F}$, then $A_{i, 0}=\mathbb{E}\left\{\lambda X_{F} X_{F}^{T}\right\}$ and $\mu_{i, 0}=\mathbb{E}\left\{\lambda X_{F}\right\}$.

Prior on $B$. Similarly, if we wish to impose a prior on $X_{B}$, then we set $A_{i, 0}=\lambda X_{B} X_{B}^{T}$ and $\mu_{i, 0}=\lambda X_{B}$. Alternatively, we can set $A_{i, 0}=\mathbb{E}\left\{\lambda X_{F} X_{F}^{T}\right\}$ and $\mu_{i, 0}=\mathbb{E}\left\{\lambda X_{F}\right\}$.

\section{RESULTS}

We expect our technique to produce transparency maps that are very similar to the ones produced by closed-form matting. The only numerical difference with closed-form results from the choice of spatial and unary priors. 


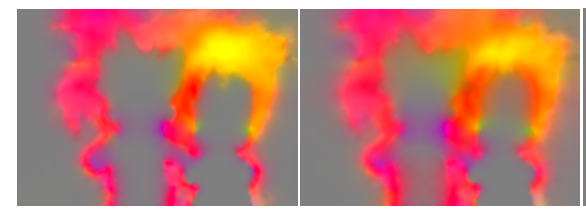

(a)

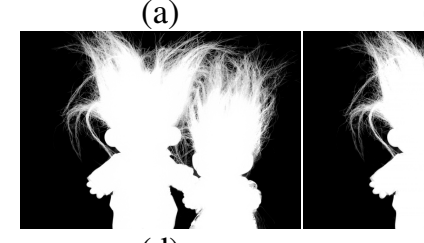

(d) (b)

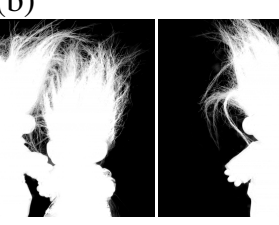

(e)

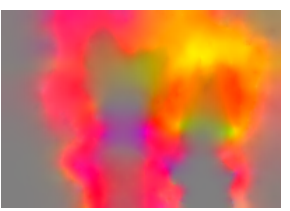

(c)

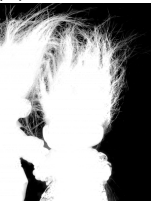

(f)
Fig. 3. Estimation at different scales. (a) $\beta$ estimated from full resolution image, $\alpha_{0}$ and confidence $\lambda$, (b) from half res, (c) from quarter res. (d) derived $\alpha$ at full res, (e) derived using upsampled $\beta$ from (f) $(\operatorname{SSIM}(\mathrm{d}, \mathrm{e})=0.9768$; $\operatorname{MAD}(\mathrm{d}, \mathrm{e})=0.0085$ ), derived using upsampled $\beta$ from (c) $(\operatorname{SSIM}(\mathrm{d}, \mathrm{f})=0.9544 ; \operatorname{MAD}(\mathrm{d}, \mathrm{f})=0.0128)$.

Although closed-form matting is being extensively used as a smoothing constraint or final filtering stage (eg. [3, 10]), it ranks poorly against the state of the art for very sparse trimaps. We propose in the following to look at a typical matting scenario where matting is first bootstrapped with a sampling step [3], which gives us an initial $\alpha_{0}$ map and confidence map $\lambda$.

\subsection{Comparison with Closed-Form Matting}

In Fig. 3, we show the difference between the $\beta$ values estimated using closed-form matting (b) and our technique (c), with $\sigma_{s}=1, \epsilon_{s}=10^{-4}$. The Mean Absolute Difference (MAD) between the derived $\alpha$ maps (e) and (f) is 0.0025 , which confirms that both approaches produce very similar results. Comparisons for the 26 examples of the training dataset of the alpha matting evaluation website [11, 12] are presented in Table 1

\subsection{Upscaling}

One key advantage of our approach is that the estimation of $\beta$ at a coarse scale can be used for estimating $\alpha$ at higher scales. In Fig. 3 we show on (a) $\beta$ estimated from a full resolution image, from half res (b) and from quarter res images (c). The derived $\alpha$ map is shown in (e) when using full res $\beta$, (f) when using upsampled half res $\beta$ and $(\mathrm{g})$ when using upsampled quarter res $\beta$. The estimated $\alpha$ maps are consistent through the scales $(\operatorname{SSIM}(\mathrm{e}, \mathrm{f})=0.9768 ; \operatorname{MAD}(\mathrm{e}, \mathrm{f})=0.0085)$ and $(\operatorname{SSIM}(\mathrm{e}$, $\mathrm{g})=0.9544 ; \operatorname{MAD}(\mathrm{e}, \mathrm{f})=0.0128)$.

Comparisons at full res, half res and quarter res for the 26 training images are reported in Table 2 and confirm that estimating $\beta$ at lower resolutions can be efficiently used for pulling an equivalent quality $\alpha$ map at full resolution.

\begin{tabular}{|c|c|c|c|}
\hline SSIM mean & SSIM std & SAD mean & SAD std \\
\hline \hline 0.9751 & 0.02 & 0.0052 & 0.0051 \\
\hline
\end{tabular}

Table 1. Difference between closed-form matting and our approach for the 26 training images of [11].

\begin{tabular}{|c|c|c|c|c|}
\hline & SSIM mean & SSIM std & SAD mean & SAD std \\
\hline \hline $1 / 2$ res & 0.9441 & 0.0437 & 0.0118 & 0.0101 \\
\hline $1 / 4$ res & 0.9138 & 0.0596 & 0.019 & 0.0165 \\
\hline
\end{tabular}

Table 2. Difference between our approach at full res and half res, full res and quarter res. The results are compiled using the 26 training images of [11].

\subsection{Running Time}

The complexity of our approach is similar to closed-form matting. The main difference is that closed-form matting requires a $5 \times 5$ stencil for $\alpha$, whereas we only need a 5 -stencil. On the other hand, in closed-form matting we only manipulate a scalar $(\alpha)$, whereas in our approach we manipulate $\beta$ and $X X^{t}$, which are of dimensions $4 \times 1$ and $4 \times 4$. In this paper, the algorithms compared are implemented in MATLAB using exact solvers and are not optimised for speed. We believe however that our approach has the potential to offer better performances as it requires less spatial access than closedform, and is also better suited for multi-grid iterative solvers because of the smoothness of $\beta$.

\subsection{Limitations}

Our method suffers from the same limitations as with closedform matting. The main issue is that the linear model of Eq. (2) is not always a good approximation. This is especially true when the background is cluttered. Also, as it is essentially a diffusion process, the geometry and sparness of the trimap has a key influence on the results. It is typically difficult to pick up isolated hair strands. Using sampling techniques as a pre-process helps reducing the issue.

\section{CONCLUSION}

We have proposed an alternative presentation for the closedform matting Laplacian. This alternative framework is built around a direct manipulation of the linear matting model parameters $a$ and $b$. The resulting transparency maps are very similar to the one obtained with closed-form matting. We have shown however that our framework yields better expositions of the spatial and unary priors and that the smoothness of the model parameters allow us to efficiently scale up transparency maps to higher resolutions. 


\section{REFERENCES}

[1] A. Levin, D. Lischinski, and Y. Weiss, "A closed-form solution to natural image matting," Pattern Analysis and Machine Intelligence, IEEE Transactions on, vol. 30, no. 2, pp. 228-242, Feb 2008.

[2] Yung-Yu Chuang, B. Curless, D.H. Salesin, and R. Szeliski, "A bayesian approach to digital matting," in Computer Vision and Pattern Recognition, 2001. CVPR 2001. Proceedings of the 2001 IEEE Computer Society Conference on, 2001, vol. 2, pp. II-264-II-271 vol.2.

[3] Kaiming He, C. Rhemann, C. Rother, Xiaoou Tang, and Jian Sun, "A global sampling method for alpha matting," in Computer Vision and Pattern Recognition (CVPR), 2011 IEEE Conference on, June 2011, pp. 2049-2056.

[4] E. Shahrian, D. Rajan, B. Price, and S. Cohen, "Improving image matting using comprehensive sampling sets," in Computer Vision and Pattern Recognition (CVPR), 2013 IEEE Conference on, June 2013, pp. 636-643.

[5] Levent Karacan, Aykut Erdem, and Erkut Erdem, "Image matting with kl-divergence based sparse sampling," June 2015.

[6] Peter M. Hillman and John M. Hannah, "Natural Image Matting," in International Conference on Video, Vision and Graphics, 2005, pp. 211-218.

[7] Kaiming He, Jian Sun, and Xiaoou Tang, "Fast matting using large kernel matting laplacian matrices," in Computer Vision and Pattern Recognition (CVPR), 2010 IEEE Conference on, June 2010, pp. 2165-2172.

[8] François Pitié and Anil C. Kokaram, "Matting with a depth map," in ICIP. 2010, pp. 21-24, IEEE.

[9] D. Singaraju, C. Rother, and C. Rhemann, "New appearance models for natural image matting," in Computer Vision and Pattern Recognition, 2009. CVPR 2009. IEEE Conference on, June 2009, pp. 659-666.

[10] Xiaowu Chen, Dongqing Zou, S.Z. Zhou, Qinping Zhao, and Ping Tan, "Image matting with local and nonlocal smooth priors," in Computer Vision and Pattern Recognition (CVPR), 2013 IEEE Conference on, June 2013, pp. 1902-1907.

[11] Christoph Rhemann, Carsten Rother, Jue Wang, Margrit Gelautz, Pushmeet Kohli, and Pamela Rott, "Alpha matting evaluation website," http://www. alphamatting.com.

[12] Christoph Rhemann, Carsten Rother, Jue Wang, Margrit Gelautz, Pushmeet Kohli, and Pamela Rott, "A perceptually motivated online benchmark for image matting," in Proceddings of the IEEE Conference on Computer Vision and Pattern Recognition, 2009, Posterpräsentation: IEEE Conference on Computer Vision and Pattern Recognition, 2009. CVPR '09, Miami, Florida, USA; 2009-06-20 - 2009-06-25. 